\title{
A dulce de leche-like product with the lipid profile of pecan oil
}

\section{Natalia Ranalli, Silvina C. Andrés, and Alicia N. Califano}

- Dulce de leche is a concentrated

sweetened milk product consumed

widely throughout Latin America.

- Unfortunately, it is high in saturated fatty acids.

- This article describes a substitute made with emulsified pecan oil that tastes like the traditional milk-based confection but is low in saturated fatty acids and high in oleic and linoleic unsaturated fatty acids.
Dulce de leche (DL), a concentrated sweetened milk product, is highly consumed in Latin America and exported to other countries, such as USA and Spain. DL is traditionally prepared by concentrating $3 \% \mathrm{w} / \mathrm{v}$ fat milk with sucrose and vanilla until $70 \%$ total soluble solids is reached, obtaining a minimum fat content of $6 \% \mathrm{w} / \mathrm{w}$. As DL is the most-consumed sweet spread in Argentina, particularly by children, it was an obvious candidate for substitution with respect to saturated fats.

The objective of this work was to improve the lipid profile of DL by replacing milk fat, which is high in saturated fatty acids (SFA), with pecan oil, which is high in unsaturated fatty acids (UFA), particularly 18:1 (52\%), $18: 2(36 \%)$, y $18: 3$ (1.5\%), leading to a DL-like product (pecan oil DL) with quality characteristics that resemble commercial DL.

Skim milk ( $0 \%$ fat) was first incubated with lactase at $4^{\circ} \mathrm{C}$ for 24 hours to reduce lactose content and subsequent crystal formation during storage. Afterward, adequate amounts of sucrose, glucose, and potassium sorbate were dissolved in the milk. At the same time, xanthan gum (0.1\%) was dissolved in a milk aliquot, and enough pecan oil to reach a level of $\sim 5 \% \mathrm{w} / \mathrm{w}$ in the final product was added, along with added tocopherols (1000 ppm) that had been pre-emulsified in another skim milk aliquot. The pecan oil DL was prepared at a La Plata University pilot plant and kept in 500g glass jars at 20ㄷ for 6 months.

DL with pre-emulsified pecan oil presented $65^{\circ}$ Brix of soluble solids, 33.6 (0.3) \% w/w moisture content, and 0.845 (0.003) water activity, while color parameters were 38.7 (1.2), 8.9 (0.1), and 19.4 (0.6) for $L^{*}, a^{*}$, and $b^{*}$, respectively. These results were in the range of com- 
mercial products. The process yield was $30.4 \%$, and according to the formula, its lipid content was $6.56 \% \mathrm{w} / \mathrm{w}$, within the range of Argentinean and Brazilian commercial DL. However, as expected, the UFA level $(89-90 \% \mathrm{w} / \mathrm{w})$-mainly consisting of oleic (C18:1 n-9 cis) and linoleic (C18:2 n-6 cis) acids-was higher, and the SFA level lower in the pecan oil DL lipid phase as compared to the FA profile of milk fat (Fig. 1). Therefore, the product presented a lower SFA/UFA ratio (0.1) with respect to the traditional product obtained with whole milk (2.1).

Pecan oil DL quality characteristics, such as physicochemical properties (aw, color, water content, crystal formation), lipid oxidation (TBARS), rheological behavior (steady state flow curves and linear oscillatory assays), typical lactose crystal formation, and microbial characterization were evaluated monthly during storage at $20^{\circ} \mathrm{C}$. TBARS values remained constant for 138 days, showing an increase afterwards (Fig. 2). However, they were not associated with sensorial changes.

In spite of the high UFA content and its high oxidation susceptibility, UFA levels did not show significant differences $(P>0.05)$ throughout storage, reflecting the antioxidant effect of the tocopherols and other natural compounds that were added to the product. Visual inspections showed that the pecan oil DL remained stable, with no oil phase separation. Moreover, in microscopic observations, no tomahawk-like shape crystals typical of $\boldsymbol{\alpha}$-lactose were detected, avoiding a characteristic defect that occurs in DL. This was probably due to a $\sim 60 \%$ of reduction in milk lactose content after lactase activity.

The pecan oil DL obtained in the pilot-plant presented the pseudoplastic flow behavior typical of DL prepared with regular milk, and within the ranges of commercial DL (Ranalli, et al. 2012). Its rheological characteristics improved during storage due to small variations, which produced a firmer structure that favored oil/water (o/w) emulsion stability. Structure development is thought to involve not only casein micelles but also

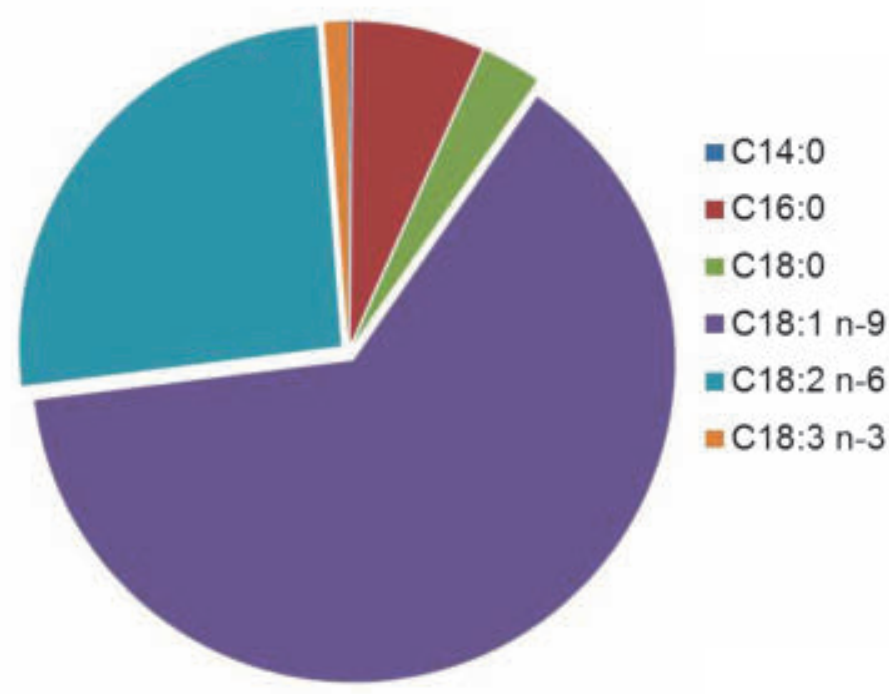

FIG. 1. Main fatty acids in pecan oil DL lipid phase

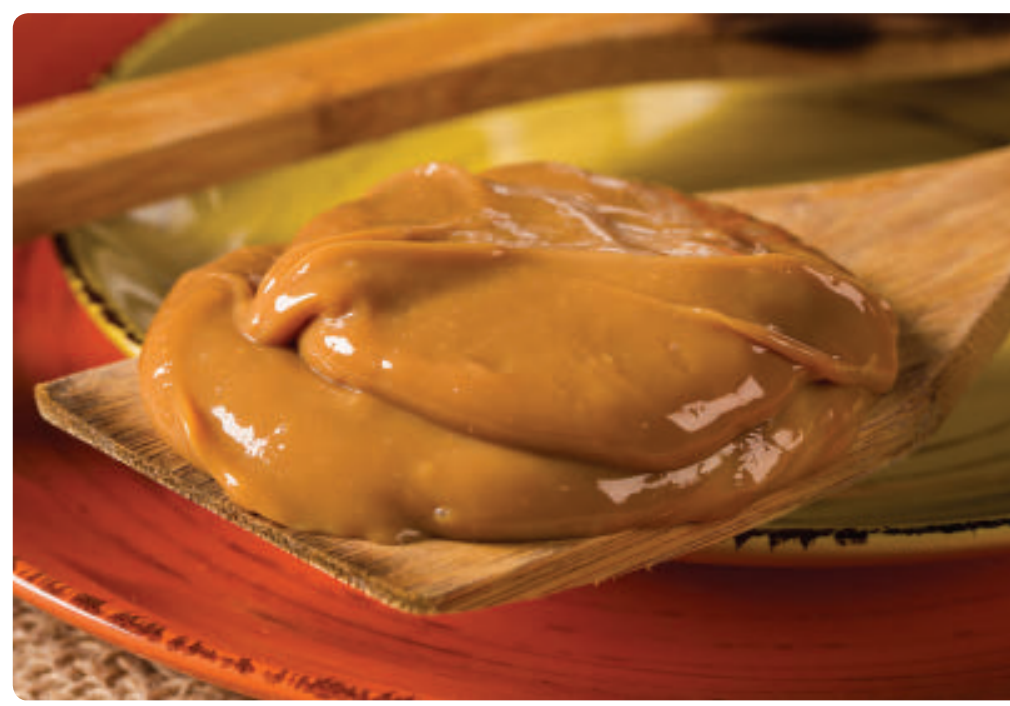

whey proteins (especially if these have suffered a high degree of denaturation during thermal processing) and fat globules. Such changes could be associated with several modifications in the continuous phase (mainly hydration of milk proteins, carbohydrates, and xanthan gum) that could happen during storage and would increase the volume fraction of dispersed components.

Molds, yeast, and coagulase positive Staphylococci counts were lower than the detection limits of the methods $(2 \log C$ $\mathrm{FUg}^{-1}$ ), indicating that thermal treatment and aseptic packaging were adequate to obtain a 6-month shelf-life.

Sensory analysis showed that more than $90 \%$ of panelists assigned more than seven points to all attributes, resulting in good product acceptability. The average scores were: $8.5(0.1)$, $8.7(0.1), 9.1(0.1)$, and $8.4(0.2)$ for general acceptability, texture, color, and taste, respectively. The pecan oil DL's caloric value was similar to traditional commercial DL. Taking the FA

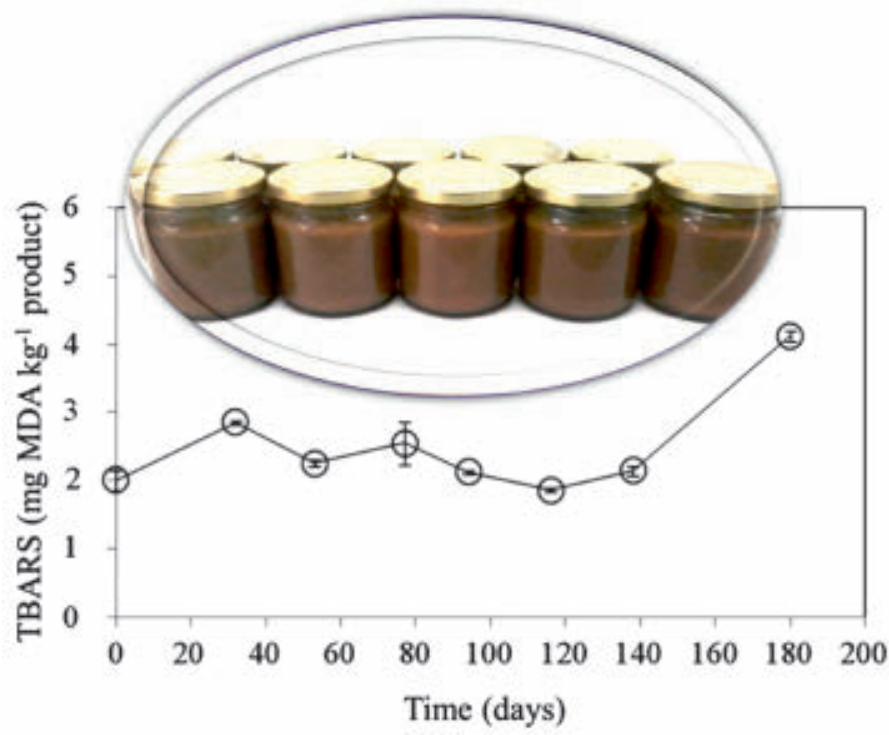

FIG. 2. TBARS evolution of pecan oil DL with added tocopherols (1000 ppm) during storage at $20^{\circ} \mathrm{C}$ 


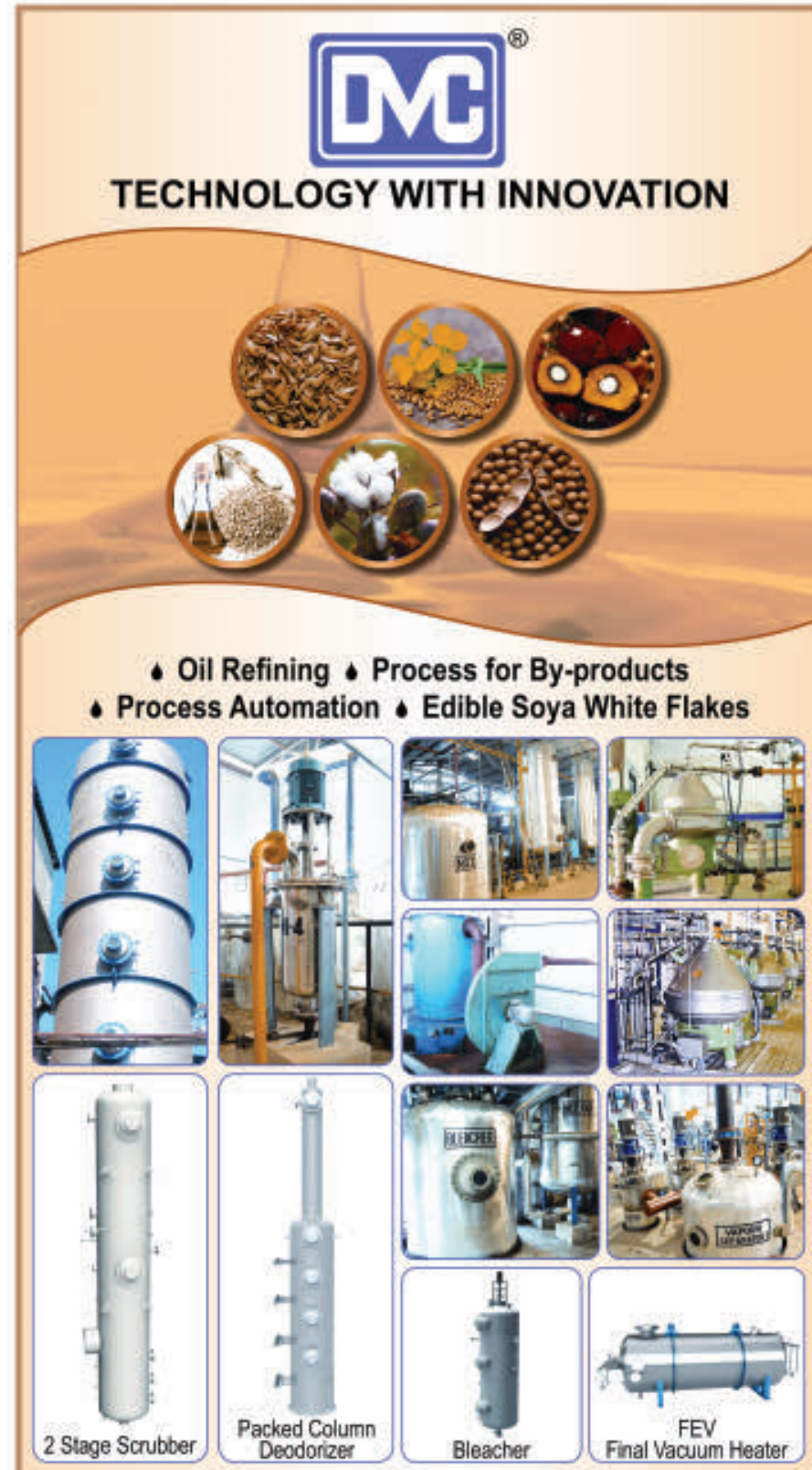

Complete turn-key solutions of Refining Vegetable Oils and Fats. Processing by-products into valuable products with the help of latest technology.

Our innovations in Edible Oil Processing:

- Use of Long-Mix De-gumming with systematic approach. - Wet Bleaching with mechanical agitation. - Inline Slurry Preparation. - No generation of flash oil in Deodorization. - Supplying cost effective and highly advanced fully automated process plants. - Development of Scrapped Surface Heat Exchanger. - Use of regenerative heat to make processes highly energy efficient.

To be a part of Innovations in Technology, Contact us :

DVC Process Technologists

DVC House, Survey No.111/11/1, Plot No.4, Opposite B.U. Bhandari Mercedes Benz Showroom, Mumbai-Bangalore Highway, Service Road,

Baner, Pune 411 045. Maharashtra (INDIA)

Phone : +912065601542 Fax : +912025893986

E-mail : sales@dvcprocesstech.com

Website : www.dvcprocesstech.com profiles of Argentinean milk fat and pecan oil into account, it could be concluded that $100 \mathrm{~g}$ of pecan oil DL would have a $82.6 \%$ lower SFA and a $200 \%$ higher UFA content than a traditional product, and it was cholesterol free.

Natalia Ranalli received her PhD degree in 2105 from La Plata National University, La Plata, Argentina, where she currently works as an assistant in the College of Engineering. For eight years before earning her doctoral degree, she worked at CIDCA (Research and Development Center on Food Cryotechnology) on healthy and nutritious processed foods development through the modification and improvement of formulations and processes involved in traditionally consumed products. She has published over four peer-reviewed international papers and two book chapters, and presented at about a dozen national and international scientific meetings. Ranalli can be contacted at nataliaranalli@gmail.com.

Silvina C. Andrés is an adjunct professor in the College of Exact Science, Department of Chemistry, La Plata National University, where she earned her Ph.D. in biochemistry in 2001. In 2005, she joined the Consejo Nacional de Investigaciones Cientificas y Técnicas (CONICET, Argentina) as a research scientist. Since 1994, she has worked in Food Chemistry and Technology at CIDCA, where she currently focuses on the physicochemical, nutritional, and sensorial quality of healthy food matrices over their shelf life. She has published over 20 peer-reviewed international papers, 5 book chapters, and 2 patents. Andrés has participated in 70 scientific meetings, presenting about 100 works.

Alicia N. Califano obtained her Ph.D. in Chemical Science in 1982 from the College of Exact Science, La Plata National University, La Plata, Argentina. That same year she became a research scientist at CONICET. Since 1989, she has worked as a full-time adjunct professor in the College of Exact Science, Department of Chemistry, La Plata National University. She has worked in Food Chemistry and Technology at CIDCA since 1977, and currently coordinates a food hydrocolloids group there. Her research focuses on design, processing, and preservation of food matrices with special nutritional characteristics, and the relationship between microstructural properties and food quality, food rheology, and food emulsions. During the last 15 years, Califano has directed or co-directed more than 15 scientific projects funded by the Ministry of Science and Technology of Argentina (MINCYT), National Council of Scientific and Technical Research (CONICET), and La Plata National University. She has published over 70 peer-reviewed international papers, 18 book chapters, and 2 patents, and has presented about 200 works at scientific meetings.

\section{References}

Ranalli, N., S.C. Andrés, and A.N. Califano, "Physicochemical and rheological characterization of "dulce de leche," J. Texture Studies 43: 115-123, 2012. 